\title{
Smart hybrid magnetic self-assembled micelles and hollow capsules
}

\author{
S. Lecommandoux ${ }^{\mathrm{a}, *}$, O. Sandre ${ }^{\mathrm{b}, *}$, F. Chécot ${ }^{\mathrm{a}}$, R. Perzynski ${ }^{\mathrm{c}}$ \\ ${ }^{a}$ Laboratoire Chimie des Polymères Organiques, Unité Mixte de Recherche 5629 Centre \\ National de la Recherche Scientifique/Ecole Nationale, Supérieure de Chimie et de \\ Physique de Bordeaux, 16, Avenue Pey Berland, 33607 Pessac, France \\ ${ }^{\mathrm{b}}$ Laboratoire Liquides Ioniques et Interfaces Chargées, Unité Mixte de Recherche 7612 Centre \\ National de la Recherche Scientifique/Université Pierre et Marie Curie 4, \\ Place Jussieu, Case 63, 75252 Paris Cedex 05, France \\ ${ }^{\mathrm{c}}$ Laboratoire Milieux Désordonnés et Hétérogènes, Unité Mixte de Recherche 7603 Centre National \\ de la Recherche Scientifique/Université Pierre et Marie Curie 4, Place Jussieu, \\ Case 78, 75252 Paris Cedex 05, France
}

\begin{abstract}
Novel magnetic nano-composites are obtained by the self-assembly in water of polypeptide-based diblock copolymers polybutadiene- $b$-poly(glutamic acid) combined with hydrophobically modified $\gamma-\mathrm{Fe}_{2} \mathrm{O}_{3}$ nanoparticles. These hybrid supramolecular objects are either filled micelles (3-d) or hollow vesicles with a magnetic membrane (2-d), deformation of which under an applied magnetic field has been evidenced. These nanoparticles are also able to respond to stimuli such as $\mathrm{pH}$ and ionic strength due to the presence of the polypeptide block, thus forming what we called multi-responsive nanocapsules. These superparamagnetic hybrid self-assemblies offer attractive potentialities in biomedicine and biotechnology due to their dimensions $(0.1-0.5 \mu \mathrm{m})$ small enough to stay for some time in the blood circulation, and due to the properties brought about by the iron oxide nanoparticles: possible manipulation by an external magnetic field gradient, local heating by a radio-frequency field for cancer radio-therapy, labeling of organs to enhance the contrast in Magnetic Resonance Imaging.
\end{abstract}

\footnotetext{
* Corresponding authors.

E-mail addresses: lecommandoux@enscbp.fr (S. Lecommandoux), osandre@enscbp.fr (O. Sandre).
} 


\section{Introduction}

High or low molecular weight synthetic amphiphiles of many types have been studied and are revealed to be interesting building blocks that can lead to highly ordered self-assembled structures in water [1]. In comparison with biological systems, depending on their molecular architecture and on external field such as temperature or $\mathrm{pH}$, numerous morphologies have now been accessed, including micelles, vesicles, tubules and more complicated morphologies $[1,2]$. The specific vesicular morphology, especially formed by the association of lipids, is one of the most interesting aspects both for material science and biological perspectives $[2,3]$. Nevertheless, applications involving these so-called liposomes are generally limited by their lack of stability probably due to a too small hydrophobic layer thickness forming the core of the liposome membrane [4,5]. Different groups reported on the vesicle formation from block copolymers (called polymersomes) in water and discussed on the improvement of the mechanical properties of the membranes obtained [6-11]. Recently, vesicles have also been obtained from peptide-based diblock copolymers in our group [12] and are called peptosomes [13,14]. In this context, the introduction of nanoscopic inorganic objects into self-organized structures is still a challenge for physico-chemists, even if Nature produces and uses frequently these hybrids. For example, calcium carbonates or phosphates [15] and iron oxides [16] are colloidal particles commonly observed in organisms that are often confined within vesicles. In addition to the biomimetic aspect of these kinds of systems, the formation of hybrids, constituted with nanoscale inorganic compounds dispersed into a self-assembled organic structure, presents two limits: the inorganic particles can be diluted and are only used as local probe, or they can really participate in the supramolecular structure and modify the resultant properties. Nevertheless, the inclusion of magnetic nanoparticles into supramolecular structures and the study of the resulting structure and its properties are still missing, even if some groups are working on the stabilization of these colloidal particles with low and/or high molecular weight surfactant, or on their introduction into polymeric gels.

A novel type of hybrid colloids is presented in this contribution, based on the association of several polymeric systems and ferrofluids. On one hand, we use inorganic nanoparticles made of magnetic iron oxide, which respond to a magnetic field of low intensity. On the other hand the organic part is made of mesoscopic structures (vesicles and micelles) self-assembled from amphiphile polybutadiene- $b$-poly(glutamic acid) diblock copolymers, conformation of which is $\mathrm{pH}$-sensitive. Those PB- $b$-PGA copolymers bearing a cross-linkable hydrophobic block and a hydrophilic peptidic block have been synthesized recently by combining anionic polymerization and ring-opening polymerization [12]. Their polydispersity indices are small enough to obtain well defined self-assembled aggregates. For example, the $\mathrm{PB}_{40}-b-\mathrm{PGA}_{100}$ copolymer leads in water to closed bilayers that also exhibit the particularity of responding to an external $\mathrm{pH}$ change by a significant variation of size: their hydrodynamic diameter $d_{\mathrm{H}}$ varies indeed from $200 \mathrm{~nm}$ in an acidic medium to $300 \mathrm{~nm}$ in a basic medium [17] (or $250 \mathrm{~nm}$ in concentrated brine). This transition with $\mathrm{pH}$ is fully reversible and is only moderately sensitive to salinity for it is not based on a simple polyelectrolyte swelling effect but on the peptidic nature of the PGA block, which exhibits a real transition between a compact helix conformation at acidic $\mathrm{pH}$ and a stretched coil conformation at basic $\mathrm{pH}$.

As for the magnetic nanoparticles, they come from ionic ferrofluids which are colloidal suspensions of nanometric magnetic grains stabilized either in aqueous media by electrostatic charges or in organic solvents by appropriate tensioactives. Before grafting these dispersant molecules, the precursor ferrofluid [18] is prepared by alkaline co-precipitation of $\mathrm{FeCl}_{2}$ and 
$\mathrm{FeCl}_{3}$ leading to $\mathrm{Fe}_{3} \mathrm{O}_{4}$ (magnetite) nanocrystals, followed by surface sign reversal with $\mathrm{HNO}_{3}$ and complete oxidation using $\mathrm{Fe}\left(\mathrm{NO}_{3}\right)_{3}$, which yields positively charged $\gamma-\mathrm{Fe}_{2} \mathrm{O}_{3}$ (maghemite) particles in $\mathrm{HNO}_{3}$. One of the goals of embedding maghemite nanoparticles into the supramacromolecular objects formed by diblock copolymers consists in utilizing the particles as probes for neutrons scattering in order to elucidate the strong morphological transformation of the vesicles during the size transition between acidic and basic $\mathrm{pH}$. One expects indeed to detect a transient opening of the bilayer and/or a leak-out of the encapsulated content. For this purpose, we benefit from the very strong contrast of the iron oxide particles compared to polymer and solvent by measuring their structure factor inside the aggregates: this $S_{\text {intra }}(q)$ gives indeed both the mean inter-particle distance [19] (hence their local concentration) and the global form factor of the object that they decorate [20]. Another guiding idea is to bring to the whole object a response to a magnetic field, either an induced shape change (ellipsoidal or not) or to trigger the delivery of an active substance by the application of a field.

\section{Results and discussion}

This work started by the verification that those organic and inorganic systems can effectively be combined together to generate well defined objects which can be properly dispersed into a solvent. Therefore we examined a series of PB- $b$-PGA copolymers which differ by the length of their polypeptide PGA block, their hydrophobic block PB being chosen to enable an easy way to cross-linking within the frame of a future freezing of the structures. In this paper, we present a few cases representative of the reachable structures:

- $\mathrm{PB}_{48}-b$ - $\mathrm{PGA}_{114}$ and $\mathrm{PB}_{48}-b-\mathrm{PGA}_{145}$ which self-assemble in water as micelles of hydrodynamic diameters $d_{\mathrm{H}}=60 \mathrm{~nm}$ and $d_{\mathrm{H}}=70 \mathrm{~nm}$, respectively, the internal diameter of the hydrophobic core being $d_{\text {int }}=14 \mathrm{~nm}$ as measured by SANS in both cases [21].

- $\mathrm{PB}_{48}-b$-PGA 56 which forms closed membranes in water, i.e. vesicles characterized by an outer diameter $d_{\mathrm{H}}=100 \mathrm{~nm}$ and a bilayer thickness $\delta=14 \mathrm{~nm}$ [21].

We tested the association of these two copolymers with three either aqueous or organic ferrofluids, in which grain size polydispersity had been reduced in a first step by the method of fractionated phase separations [22] by adding excess $\mathrm{HNO}_{3}$ :

- $\mathrm{S} 1 \mathrm{~S}-\mathrm{HNO}_{3}$ acidic ferrofluid is an aqueous acidic ferrofluid $(\mathrm{pH}=1.2)$ with a particle size distribution characterized by a Log-normal law of parameters, $d_{0}=6.6 \mathrm{~nm}$ and $\sigma=0.21$. Its iron oxide surface is coated simply by $\mathrm{H}^{+}$protons, thereby leaving a free access for a future grafting by the glutamic acid moieties of the copolymers.

- S1S-Na ${ }_{3}$ Cit citrated ferrofluid comes from the previous acidic ferrofluid after coating by trisodium citrate ligands, thus enabling a dispersion at $\mathrm{pH}=7$.

- S2- $\mathrm{CH}_{2} \mathrm{Cl}_{2}$ surfacted ferrofluid is grafted by a phosphoric di-ester type tensioactive (Beycostat NE) for a solubilization in dichloromethane. It also has a slightly broader distribution of particle sizes $\left(d_{0}=6.8 \mathrm{~nm}, \sigma=0.24\right)$.

The assembly of each copolymer mixed with either 1 or 2 equivalents of the surfacted ferrofluid $\mathrm{S} 2-\mathrm{CH}_{2} \mathrm{Cl}_{2}$ was favored by first eliminating the dichloromethane and then redispersing the objects into water, after deprotonating the acidic groups of the copolymer with $\mathrm{NaOH}$ to raise the $\mathrm{pH}$ up to $\approx 7$. In the case of the bare acidic particles $\mathrm{S} 1 \mathrm{~S}-\mathrm{HNO}_{3}$, the flocculation was 
induced almost instantaneously after the addition of PB- $b$-PGA copolymers. The supernatant could then be easily replaced by $\mathrm{CH}_{2} \mathrm{Cl}_{2}$. Finally with $\mathrm{S} 1 \mathrm{~S}-\mathrm{Na}_{3} \mathrm{Cit}$ ferrofluid, the interaction with all the polymers did not lead to any visible destabilization.

After three weeks, all suspensions were analyzed by dynamic light scattering (DLS) and small angle neutrons scattering [23] (SANS). Thus we could show that some of the (co)polymerparticle aggregates reach an equilibrium shape and a proper dispersion state.

Samples $\mathrm{PB}_{48}-b$ - $\mathrm{PGA}_{114}$ and $\mathrm{PB}_{48}-b$ - $\mathrm{PGA}_{145}$ mixed with the $\mathrm{S} 2-\mathrm{CH}_{2} \mathrm{Cl}_{2}$ surfacted ferrofluid (A) leads to a dispersion of magnetic micelles in water, diameters $d_{\mathrm{H}}$ of which are between $430 \mathrm{~nm}$ and $225 \mathrm{~nm}$ (Tables 1 and 2). The large increase of the outer diameter compared to unloaded micelles $\left(d_{\mathrm{H}}=60 \mathrm{~nm}\right.$ and $70 \mathrm{~nm}$ for $\mathrm{PB}_{48}-b-\mathrm{PGA}_{114}$ and $\mathrm{PB}_{48}-b$ - $\mathrm{PGA}_{145}$, respectively [21]) suggests that their hydrophobic cores are filled with nanoparticles at a high local concentration. The short inter-particle distance given around $80 \AA$ by the position of the structure peak (Fig. 1) corresponds to a volume fraction of nanoparticles inside the micelles of about $45 \%$, of the order of the dense packing value. This high encapsulation yield together with the global 3-d shape of the aggregates is confirmed by a look at the TEM and AFM images in the case of $\mathrm{PB}_{48}-b$-PGA 114 , which shows large and thick baggies of inorganic particles. Please note that the copolymer samples combined with 2 mass equivalents of ferrofluid lead to final volume fractions $\Phi$ of nanoparticles (from titration of iron) which are 6-7 times smaller than those with 1 mass equivalent (Fig. 2). This difference originates from the fact that the objects are fully dispersed only in the former case but not in the latter case. Thus with 2 equivalents, we get supernatants which are globally more dilute in copolymer-particles complexes.

Table 1

Short summary of samples and self-assembled hybrid objects that have been prepared

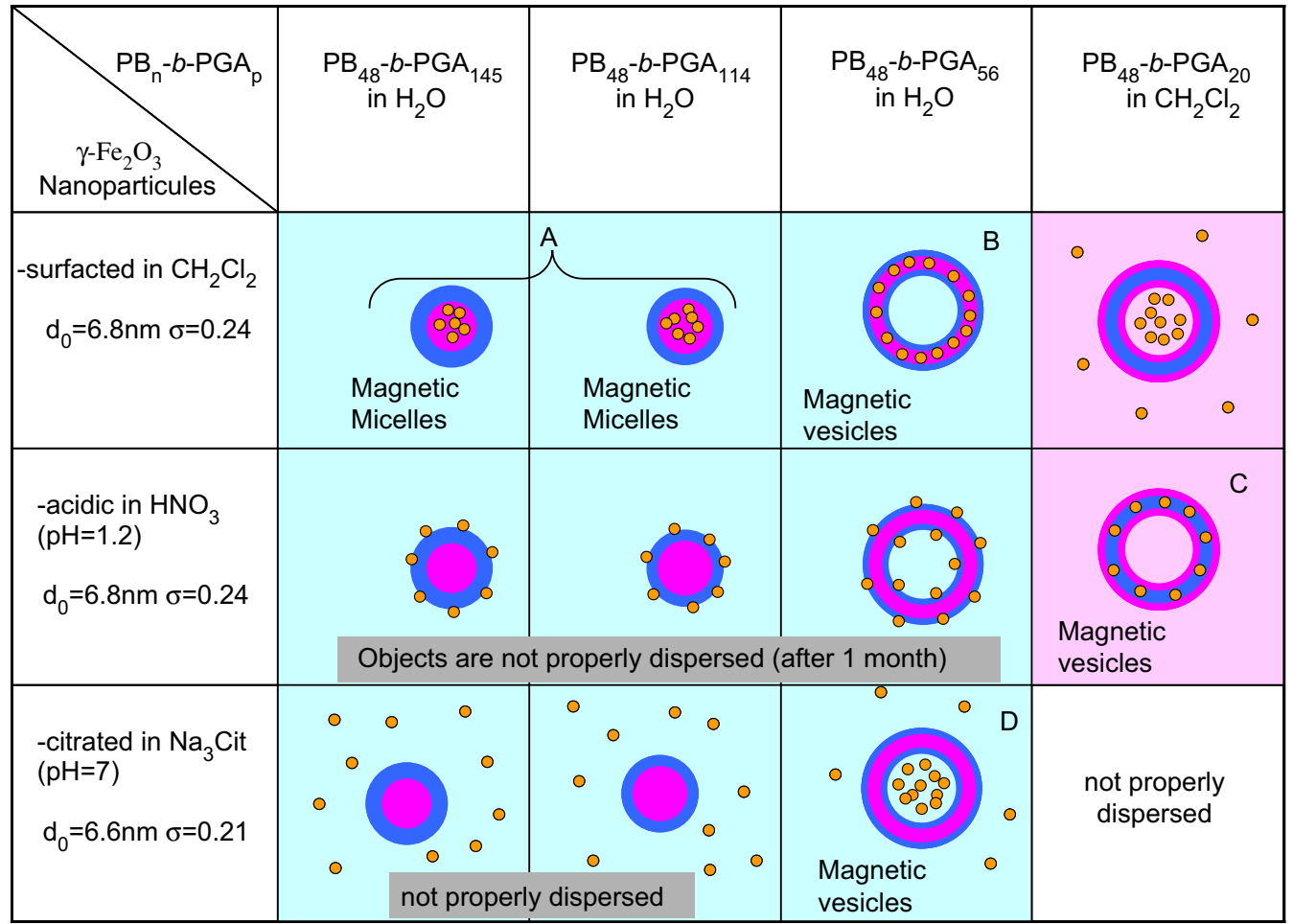


Table 2

Characterization of the systems under study (real concentration in ferrofluid, size and shape)

\begin{tabular}{llllllll}
\hline Copolymer & Ferrofluid & Eq. ferro & Solvent & System $^{\mathrm{b}}$ & $\Phi_{\text {ferro }}(\%)^{\mathrm{c}}$ & $d_{\mathrm{H}}(\mathrm{nm})^{\mathrm{d}}$ & Morphology \\
\hline $\mathrm{PB}_{48}-b-\mathrm{PGA}_{114}$ & $\mathrm{~S} 2-\mathrm{CH}_{2} \mathrm{Cl}_{2}$ & 1 & $\mathrm{H}_{2} \mathrm{O}$ & $\mathrm{A}$ & 0.14 & 333 & Micelle \\
& & 2 & & $\mathrm{~A}$ & 0.021 & 260 & Micelle \\
$\mathrm{PB}_{48}-b-\mathrm{PGA}_{145}$ & $\mathrm{~S} 2-\mathrm{CH}_{2} \mathrm{Cl}_{2}$ & 1 & $\mathrm{H}_{2} \mathrm{O}$ & $\mathrm{A}$ & 0.26 & 429 & Micelle \\
& & 2 & & $\mathrm{~A}$ & 0.043 & 225 & Micelle \\
$\mathrm{PB}_{48}-b-\mathrm{PGA}_{56}$ & $\mathrm{~S} 2-\mathrm{CH}_{2} \mathrm{Cl}_{2}$ & 1 & $\mathrm{H}_{2} \mathrm{O}$ & $\mathrm{B}$ & 0.30 & 624 & Vesicle \\
& & 2 & & $\mathrm{~B}$ & 0.26 & 208 & Vesicle \\
$\mathrm{PB}_{48}-b-\mathrm{PGA}_{20}$ & $\mathrm{~S} 1 \mathrm{~S}-\mathrm{HNO}_{3}$ & 2 & $\mathrm{CH}_{2} \mathrm{Cl}_{2}$ & $\mathrm{C}$ & 0.13 & $466 / 76^{\mathrm{e}}$ & Micelle + vesicle \\
& $\mathrm{S} 2-\mathrm{HNO}_{3}$ & 2 & & $\mathrm{C}$ & 0.25 & 68 & Micelle \\
$\mathrm{PB}_{48}-b-\mathrm{PGA}_{56}$ & $\mathrm{~S} 1 \mathrm{~S}-\mathrm{Na}_{3} \mathrm{Cit}$ & 2 & $\mathrm{H}_{2} \mathrm{O}$ & $\mathrm{D}$ & 6.05 & - & Vesicle \\
\hline
\end{tabular}

${ }^{a}$ Mass equivalent of ferrofluid compared to the copolymer in preparation.

b Code corresponding of the system related to Table 1 and to the text.

c Real concentration of ferrofluid in the final mixture (measured by titration of iron).

d Hydrodynamic diameter as measured by DLS with CONTIN analysis.

e Two populations were observed by DLS.

These peptide-based micellar systems are thus able to encapsulate a large volume of nanoparticles, inducing an over-concentration in these baggies (about 30\%), even if the global concentration in the solution is very low $\left(\Phi_{\text {ferro }} \approx 0.1 \%\right)$. More interestingly, these systems are pretty stable compared to ferrofluids alone at the same concentration. Another interesting point comes from the fact that these diblocks are able to be stabilized in micelles with very big sizes when loaded with nanoparticles compared to their equilibrium state in pure water solutions.

Vesicles of $\mathrm{PB}_{48}-b$ - $\mathrm{PGA}_{56}$ still form in water when in the presence of $\mathrm{S} 2-\mathrm{CH}_{2} \mathrm{Cl}_{2}(\mathbf{B})$, their outer diameter being significantly increased $\left(d_{\mathrm{H}}=620 \mathrm{~nm}\right.$ and $210 \mathrm{~nm}$ for 1 and 2 mass equivalents, respectively). Due to their hydrophobicity, the surfacted nanoparticles are confined in 2-d between the two leaflets of the copolymer bilayer, as proved separately by SANS

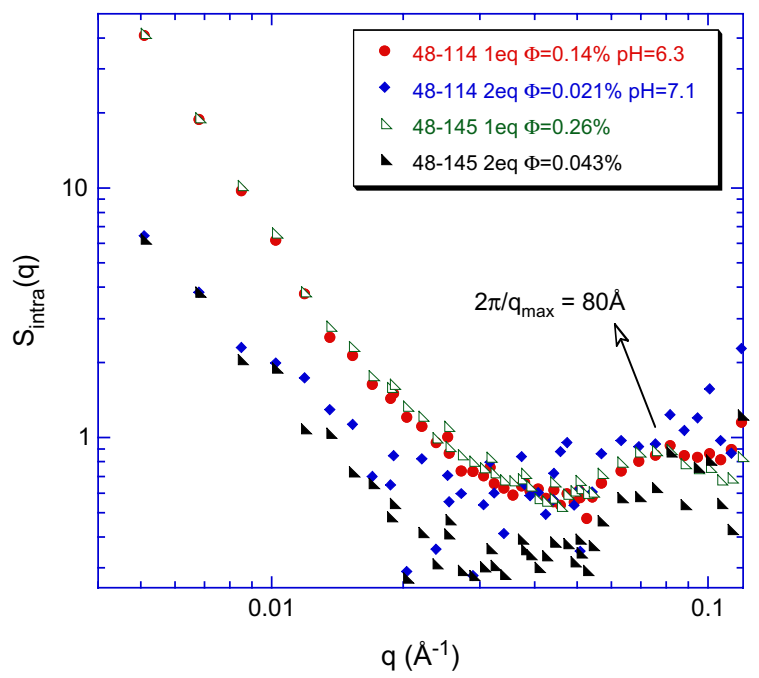

Fig. 1. Intra-aggregates' structure factor $S_{\text {intra }}(q)$ for hydrophobic nanoparticles associated to $\mathrm{PB}_{48}-b$ - $\mathrm{PGA}_{114}$ and $\mathrm{PB}_{48}-b-\mathrm{PGA}_{145}$, as measured by SANS [24]. 


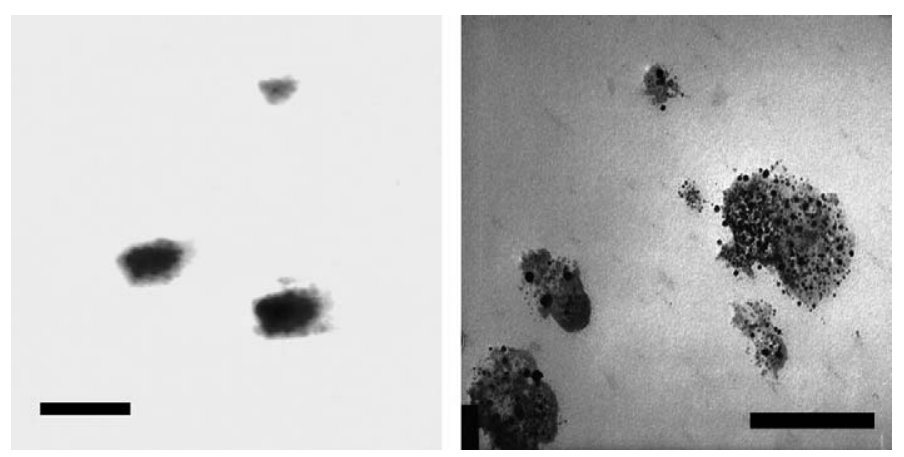

Fig. 2. AFM (left) and TEM (right) pictures of hybrid copolymer micelles loaded with magnetic nanoparticles, obtained by combining $\mathrm{PB}_{48}-b-\mathrm{PGA}_{114}$ and 1 mass equivalent of surfacted ferrofluid $\mathrm{S} 2-\mathrm{CH}_{2} \mathrm{Cl}_{2}$. The scale bar measures $400 \mathrm{~nm}$ in both cases.

measurements, TEM pictures and AFM imaging (which in addition contains a valuable topographical information). Fig. 3 represents the intra-aggregate structure factor $S_{\text {intra }}(q)$ measured by SANS for the hydrophobic nanoparticles associated to $\mathrm{PB}_{48}-b-\mathrm{PGA}_{56}$ diblock copolymer forming vesicles. In the small $q$-regime, the form factor of the aggregate follows a power law with a slope approximately-2 typical for flat samples, thus confirming a vesicle-type morphology of the organic-inorganic complex. In this $q$-region, one can calculate the thickness of the layer forming the vesicle membrane $\left(\log q^{2} S_{\text {intra }}(q) \approx-q^{2} \delta^{2} / 12\right)$. From these data, we obtained $\delta=8.6 \mathrm{~nm}$ and $\delta=17.7 \mathrm{~nm}$, respectively, for the system with 1 equivalent and 2 equivalent of the ferrofluid. As the diameter of the ferrofluid itself is $6.8 \mathrm{~nm}$, one can conclude that this membrane is formed by one or two layers of the magnetic colloids depending on the preparation. These results corroborate the fact that the structure peak due to the particles in contact $(8 \mathrm{~nm})$ is absent for the preparation obtained with 1 equivalent of ferrofluid that only forms one layer of the inorganic colloid inside the polymeric membrane.
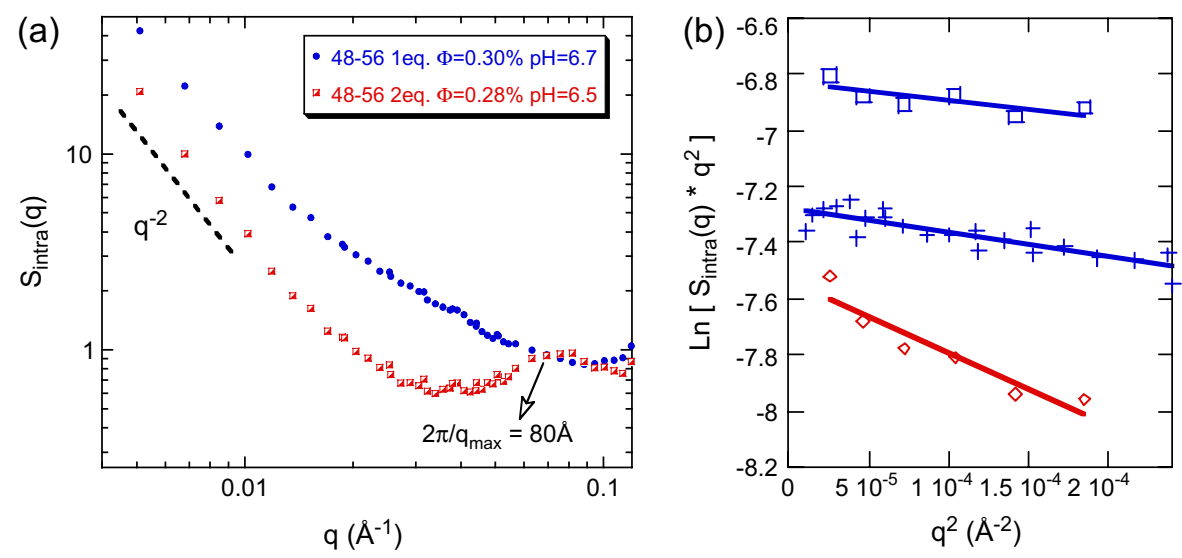

Fig. 3. (a) Intra-aggregates' structure factor $S_{\text {intra }}(q)$ for hydrophobic nanoparticles $\mathrm{S}_{2}-\mathrm{CH}_{2} \mathrm{Cl}_{2}$ associated to $\mathrm{PB}_{48}-b$ PGA $_{56}$, as measured by SANS [24]. (b) Kratky-Porod plots of the same data, slope of which gives the values $\delta_{1 \mathrm{eq}}$ and $\delta_{2 \mathrm{eq}}$ of membrane thickness. 
(a)

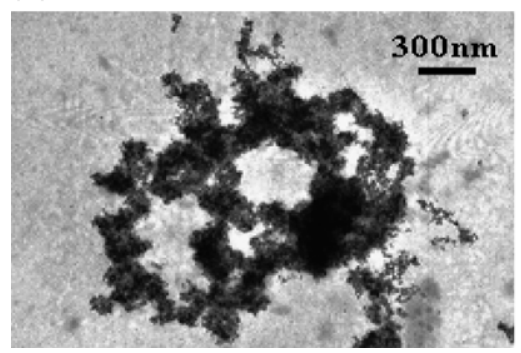

(b)

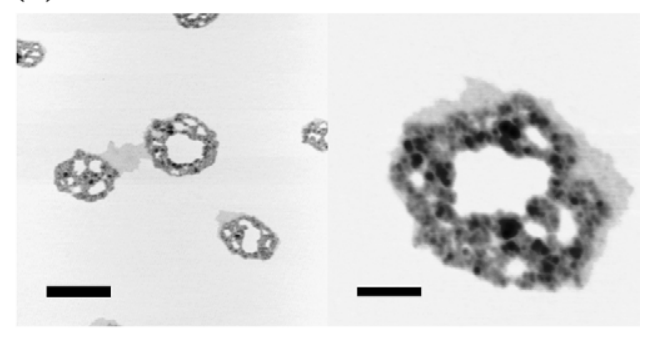

Fig. 4. (a) TEM picture of a magnetic membrane made of $\mathrm{PB}_{48}-b-\mathrm{PGA}_{56}$ and 1 equivalent of $\mathrm{S} 2-\mathrm{CH}_{2} \mathrm{Cl}_{2}$ (bar length is $333 \mathrm{~nm}$ ); (b) AFM pictures of the same sample (the bar measures $500 \mathrm{~nm}$ on the left and $150 \mathrm{~nm}$ on the right).

These data are confirmed by TEM (Fig. 4a) and AFM (Fig. 4b) observations done on the same samples. TEM and AFM measures show only the nanoparticles, respectively, because of the low electron density and low mechanical properties of the polymer compared to the iron oxide. The uniform film visible around the objects by AFM is likely the pure copolymer bilayer spreading onto the mica substrate, with a measured height $\delta_{\mathrm{AFM}}=4.5 \mathrm{~nm}$. The vesicles appear holey on the pictures because the strong adhesion of those soft shells on high energy surfaces (freshly cleaved mica for AFM or Formvar coated graphite for TEM) necessitates the ripping of the bilayers (a sort of like peeled orange skins!).

To the authors' knowledge this is the first described case of vesicles with a fluid magnetic membrane [25]. Several magnetic shells were already mentioned in literature [26,27], but none as hollow and floppy as ours. An appropriately designed theory describing their deformation under an applied magnetic field predicts a prolate-oblate transition in that case [28].

Vesicles and micelles of $\mathrm{PB}_{48}-b$ - $\mathrm{PGA}_{20}$ can also be obtained in $\mathrm{CH}_{2} \mathrm{Cl}_{2}$ depending on the hydrophilic magnetic particles used $(\mathbf{C})$. When 2 equivalents of the $\mathrm{S} 1 \mathrm{~S}-\mathrm{HNO}_{3}$ ferrofluid are added to $\mathrm{PB}_{48}-b$ - $\mathrm{PGA}_{20}$ the system tends to form a mixture of micelles and vesicles with $d_{\mathrm{H}}$, respectively, of $76 \mathrm{~nm}$ and $466 \mathrm{~nm}$, as can be observed by DLS. When the other ferrofluid $\mathrm{S} 2-\mathrm{HNO}_{3}$ is added to $\mathrm{PB}_{48}-b-\mathrm{PGA}_{20}$ the equilibrium structure becomes only spherical micelles with a $d_{\mathrm{H}}=68 \mathrm{~nm}$. As the two ferrofluids used are almost identical in terms of size and polydispersity, this difference traduces that the vesicle morphology is probably not thermodynamically stable under these experimental conditions. Moreover, the interaction of the magnetic colloids with the copolymer seems also to be quite different, resulting in a different volume ratio in the solution.

Vesicles self-assembling in water from $\mathrm{PB}_{48}-b$ - $\mathrm{PGA}_{56}$ can also be filled in their inner aqueous compartment (D) with a neutral ferrofluid just as $\mathrm{S} 1 \mathrm{~S}-\mathrm{Na}_{3} \mathrm{Cit}$, thereby obtaining magnetic polymersomes analogous to magnetoliposomes [29], which are lipid vesicles loaded with an aqueous ferrofluid at $\mathrm{pH}$ 7. In the same way as for magnetic liposomes, after preparing the vesicles one can separate them from nonencapsulated nanoparticles by column chromatography with Sephacryl S1000 as a separating medium [29]. The sorting process is studied by a look at the structure factor $S_{\text {intra }}(q)$.

The low $q$-region of Fig. 5 is a part of the form factor of large 3-d objects present in both samples. The structure peak in the initial unsorted dispersion gives a local concentration $\Phi_{\text {local }}=11 \%$ inside the vesicles superior to the average concentration $\Phi=6 \%$ (from iron titration). The less pronounced shoulder is still visible in the curve after passage through the column corresponds to $\Phi_{\text {local }}=0.07 \%$, which is 3-4 times higher than the average $\Phi=0.02 \%$. The 


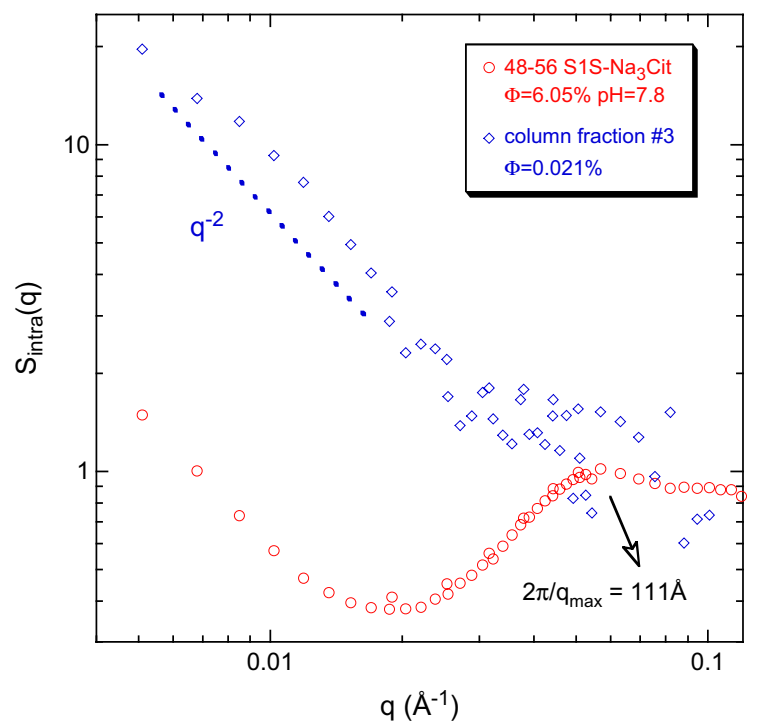

Fig. 5. SANS curves for $\mathrm{PB}_{48}-b$ - $\mathrm{PGA}_{56}$ vesicles prepared in the citrated ferrofluid S1S-Na ${ }_{3}$ Cit before and after column fractionation.

enrichment of loaded vesicles compared to free particles is thus concomitant with a global dilution of all the objects and a partial leak-out of the vesicles, maybe due to a larger size of copolymer vesicles than liposomes described in Ref. [29], compared to the porosity of the separating medium.

\section{Conclusions}

The main challenge addressed in this paper was to build complexes of maghemite nanoparticles and diblock copolymers into supra-macromolecular objects. The use of these inorganic magnetic particles had two advantages: the first one resides in the fact that they can be model systems for encapsulation of hydrophilic and/or hydrophobic species; the second one consists in utilizing the particles as probes for neutrons scattering in order to elucidate the strong morphological transformation of the vesicles or micelles that were formed by the organic material itself. We demonstrated that spherical micelles in water filled with hydrophobic ferrofluid can be stabilized at a concentration as high as $45 \%$ (volume concentration in the micelles). The vesicular morphology is even more interesting than the micelles: these hollow baggies can either encapsulate isolated volumes of hydrophilic ferrofluid or, when the nanoparticles are on the contrary hydrophobic, confine them within the membranes, leading to 2-dimensional "necklace" structure observed by TEM. Finally, inverse systems in organic solvent (dichloromethane) can be obtained by forming spherical micelles or mixtures of micelles and vesicles depending on the hydrophilic ferrofluid used.

Now that we elucidated the self-assembly of those 2-d or 3-d hybrid objects made of magnetic nanoparticles and (co)polymers, the next steps of this study will consist in attempting to modify their shape thanks to the magnetic field. We have started by studying the deformation of the vesicles confining maghemite nanoparticles within their membrane under a constant 
magnetic field B [30]: in that case, the anisotropy of the SANS patterns demonstrates a local reorganization of the nanoparticles attributed to an overall ellipsoidal deformation of the vesicles. Then with a time varying field B (for instance a rotating field) we could generate further complex shapes like the starfish-like ones well known for concentrated ferrofluid droplets. Preparing those original shapes with samples at the mesoscopic scale represents a challenge for the physical chemistry community. The aggregates of ferrofluids with diblock rod-coil copolymers open a possible route to reach this goal, also offering the possibility to freeze the structures by cross-linking unsaturated blocks like polybutadiene. The deformation of soft magnetic objects by appropriate DC or AC fields followed by cross-linking seems to us a novel and original way to reach complex mesoscopic shapes. In addition and even more interestingly, these magnetic nanocapsules that can be filled either by hydrophobic and hydrophilic species certainly represent a very promising class of materials for encapsulation of various drugs. By combining a $\mathrm{pH}$ change with the application of a magnetic field, we could further enhance the deformation of those vesicles and deliver drugs more efficiently than with a single stimulus. We are currently working in these directions.

\section{References}

[1] Riess G. Prog Polym Sci 2003;28:1107.

[2] For a review on polymer vesicles, see Discher DE, Eisenberg A. Science 2002;297:967.

[3] Lipowsky R, Sackmann E. In: Structure and dynamics of membranes. New York: Elsevier; 1995.

[4] Lasic DD, Papahadjopoulos D. In: Medical applications of liposomes. New York: Elsevier; 1998.

[5] Marsh D. CRC handbook of lipid bilayers. Boca Raton, FL: CRC Press; 1990.

[6] Cornelissen JJLM, Fischer M, Sommerdijk NAJM, Nolte RJM. Science 1998;280:1427.

[7] Discher BM, Won Y-Y, Ege DS, Lee JC-M, Bates FS, Discher DE, et al. Science 1999;284:1143.

[8] Aranda-Espinoza H, Bermudez H, Bates FS, Discher DE. Phys Rev Lett 2001;87:208301.

[9] Lee JC-M, Santore M, Bates FS, Discher DE. Macromolecules 2002;35:323.

[10] Dimova R, Seifert U, Pouligny B, Forster S, Döbereiner H-G. Eur Phys J E 2002;7:241.

[11] Bermudez H, Brannan AK, Hammer DA, Bates FS, Discher DE. Macromolecules 2002;35:8203.

[12] Chécot F, Lecommandoux S, Gnanou Y, Klok H-A. Angew Chem Int Ed 2002;41:1340.

[13] Kimura S, Kim DH, Sugiyama J, Imanishi Y. Langmuir 1999;15:4461.

[14] Kukula H, Schlaad H, Antonietti M, Forster S. J Am Chem Soc 2002;124:1658.

[15] Mann S. Angew Chem Int Ed 2000;39:3393.

[16] Chasteen ND, Harrison PM. J Struct Biol 1999;126:182.

[17] Chécot F, Lecommandoux S, Klok H-A, Gnanou Y. Eur Phys J E 2003;10:25.

[18] Massart R. IEEE Trans Magn 1981;17:1247.

[19] Dubois E, Boué F, Cabuil V, Perzynski R. J Chem Phys 1999;111:7147.

[20] Oberdisse J, Demé B. Macromolecules 2002;35:4397.

[21] Chécot F, Brûlet A, Oberdisse J, Gnanou Y, Mondain-Monval O, Lecommandoux S. Langmuir 2005;21:4308.

[22] Massart R, Dubois E, Cabuil V, Hasmonay E. J Magn Magn Mater 1995;149:1.

[23] Sandre O, Chécot F, Lecommandoux S, Perzynski R, Oberdisse J. Experiment 6899. PACE spectrometer, Laboratoire Léon Brillouin, CEA-Saclay: France; 2003.

[24] Intra-aggregate structure factor $\operatorname{Sintra}(Q)$ for nanoparticles associated to the block copolymers has been measured by SANS from the scattered intensity of the particle-copolymer complexes normalized by the volume fractions $\Phi$ of particles and divided by the form factor $P(Q)$ of the ferrofluid alone.

[25] Lecommandoux S, Sandre O, Chécot F, Rodriguez-Hernandez J, Perzynski R. Adv Mater 2005;17:712.

[26] Bizdoacaa EL, Spasovaa M, Farlea M, Hilgendorff M, Caruso F. J Magn Magn Mater 2002;240:44.

[27] Shchuki DG, Sukhorukov GB, Möhwald H. Angew Chem Int Ed 2003;42:4472.

[28] Kern N, Fourcade B. Europhys Lett 1997;38:395.

[29] Lesieur S, Grabielle-Madelmont C, Menager C, Cabuil V, Dadhi D, Pierrot P, et al. J Am Chem Soc 2003; $125: 5266$.

[30] Lecommandoux S, Sandre O, Chécot F, Rodriguez-Hernandez J, Perzynski R. Adv Mater 2005;17:712. 